COMMUNICATIONS IN

ANALYSIS AND GEOMETRY

Volume 12, Number 5, 1155-1171, 2004

\title{
On a Multi-particle Moser-Trudinger Inequality
}

\author{
HAO FANG
}

\begin{abstract}
We verify a conjecture of Gillet-Soulé. We prove that the determinant of the Laplacian on a line bundle over $\mathbb{C P}^{1}$ is always bounded from above. This can also be viewed as a multi-particle generalization of the Moser-Trudinger Inequality. Furthermore, we conjecture that this functional achieves its maximum at the canonical metric. We give some evidence for this conjecture, as well as links to other fields of analysis.
\end{abstract}

\section{Introduction.}

For a compact complex curve $C$ with a Hermitian metric, $g^{C}$, and a line bundle $l$ over $C$, with associated Hermitian metric, $g^{l}$, the determinant of the $\bar{\partial}$-aplacian over $\mathcal{O}(l)$ is defined by the method of zeta-regularization. We denote its logarithm as $A\left(g^{C}, g^{l}\right)$, a functional of $g^{C}$ and $g^{l}$.

In [3], Gillet and Soulé posed the following:

Conjecture 1.1 (Gillet-Soulé). $A\left(g^{C}, g^{l}\right)$ is bounded from above by a constant independent of choice of $g^{C}$ and $g^{l}$.

Conjecture 1.1 is motivated by the Arithmetic Riemann-Roch theorem; its bounded-ness will imply the one-side bounded-ness of the modified arithmetic Betti number, first introduced by Gillet and Soulé. We point out that the original conjecture of [3] is more general; however, we only concentrate on this simple case in this paper.

From an analytic point of view, $A\left(g^{C}, g^{l}\right)$ is a spectral invariant that is naturally linked to the quantization problem of Toeplitz operators. The conjecture can also be viewed a natural holomorphic extension of the classical Szegö limit theorem, which was originally stated for $S^{1}$ [4]. See Section 5 for more details.

On each smooth closed curve $C$, there exists a canonical metric with constant curvature, $g_{0}^{C}$, which in turn induces a canonical metric on $l, g_{0}^{l}$. By the Uniformization Theorem, general metrics on $C$ and $l$ are conformal to $g_{0}^{C}$ and $g_{0}^{l}$, respectively. According to the Serre Duality, one only needs to consider ample line bundles over the curve; hence, it also can be interpreted 
as an analytic torsion associated to the line bundle $l$ (See Section 2 for more details). A simple application of the family Riemann-Roch theorem of Bismut-Gillet-Soulé [1] then gives the anomaly formula of the regularized determinant in terms of the anomaly of the Quillen metric and the $\mathrm{L}^{2}$-metric. Furthermore, by a similar computation, $g^{C}$ can be fixed (see [3] for details); hence, it is enough to prove the conjecture for $g^{C}=g_{0}^{C}$ and $g^{l}=\exp \varphi g_{0}^{l}$.

In the same paper, Gillet and Soule considered the case of $C$ being rational. They proved the conjecture under the condition that the metric $g^{l}$ is endowed with an additional rotational symmetry. The proof is a delicate extension of the original proof of Moser for the famous Moser-Trudinger Inequality for two-sphere [9].

In this paper, we remove the special condition and prove the conjecture for the rational curve case in general:

Theorem 1.2. Conjecture 1.1 holds for any holomorphic line bundle over $\mathbb{C P}^{1}$.

Naturally, we consider the problem of exact upper bound. We conjecture that

Conjecture 1.3. $A\left(g_{0}^{C}, g^{l}\right)$ achieves its sharp upper bound only when $g^{l}$ is the standard metric $g_{0}^{l}$.

See Conjecture 5.2 for an equivalent but more concrete statement.

We have some partial results verifying Conjecture 1.3 in special cases: It holds for a function $\varphi$ with large $\mathrm{L}^{2}$ norm (see Theorem 3.2); also, constant functions are local maximal points of the determinant functional $A\left(g_{0}^{C}, g^{l}\right)$; i.e., Conjecture 1.3 holds for function $\varphi$ with very small energy.

In this paper, we will give several interpretations of this conjecture.

Firstly, it can be viewed as sharp form of a multi-particle generalization of the Moser-Trudinger inequality [9]. In fact, Theorem 1.2 can be viewed as the first step of an attempt to emulate Moser's original proof of the original Moser-Trudinger Inequality; the special case solved by Gillet and Soulé in [3] is a delicate extension of the Moser's original approach. We remark that Jost-Wang considered a different type of extension of the Moser-Trudinger inequality relating to the Toda system [7].

Secondly, it is a $S^{2}$ holomorphic extension of the classical Szegö limit theorem, which was stated for $S^{1}$ in the frame work of Fourier analysis. Notice that Okikiolu extended a weak form of Szegö limit theorem to $S^{2}$ and $S^{3}$ [10]. However, our conjecture is a sharp inequality for all finite 
natural number $n$, and we consider a holomorphic version. See Section 5 for more details.

Thirdly, it can be viewed as a problem related to the quantization of the Toeplitz operator on $S^{2}$. It is thus closely related to earlier works of Boutet de Monvel-Guillemin [2] and Uribe [13].

Finally, it relates to a limit theorem when interpreted as a configuration problem of random variables on $S^{2}$. This is partly inspired by the Kac's probabilistic approach to the Szegö limit theorem [8], and its generalization by Johansson [6].

This paper is organized as follows. In Section 2, we set up the problem and state the result of Gillet-Soulé. In Section 3, we prove Theorem 1.2. In Section 4, we establish the local maximality of the constant function for the log determinant functional. In Section 5, we pose the new conjecture on the sharp bound and give several formulations of the problem, relating it to different classical results.

Acknowledgement The author would like to thank Paul Yang for bringing this problem to his attention. Thanks are also due to Sun-Yung Chang, Xianzhe Dai, Kate Okikiolu and Paul Yang for discussions. Part of this work was done during the author's stay in MSRI in 2001.

\section{Set-up.}

In this Section, we define the related geometric objects and state the previous results of [3].

From now on, we fix $C=\mathbb{C P}^{1}=\mathbb{C} \cup\{\infty\}$ with $z$ being the complex coordinate function. Let

$$
\mu=\frac{\sqrt{-1} d z d \bar{z}}{2 \pi\left(1+|z|^{2}\right)^{2}}
$$

be the Kähler form of the standard Fubini-Study metric such that $\int_{C} \mu=1$.

Notice that, with our notation, the celebrated Moser-Trudinger Inequality reads as $[9]$

$$
\log \int_{C} \exp (\varphi) \mu-\frac{1}{4} \int_{C}|\nabla \varphi|^{2} \mu-\int_{C} \varphi \mu \leq 0 .
$$

Fix a positive integer $n$. $\mathcal{O}(n)$ denotes the holomorphic line bundle over $C$ of degree $n$. For a real $C^{\infty}$ function $\varphi$ over $C$, we can define the following metric $g_{\varphi}$ on $\mathcal{O}(n)$ :

$$
<s, t>_{\varphi}=\int_{C} \frac{s \bar{t}}{\left(1+|z|^{2}\right)^{n}}(\exp \varphi) \mu
$$


where $s$ and $t$ are two sections of $\mathcal{O}(n)$. We then have the relation $g_{\varphi}=$ $(\exp \varphi) g_{0}$.

We consider the following chain complex

$$
0 \rightarrow \Lambda^{0,0}(C, \mathcal{O}(n)) \stackrel{\bar{\partial}}{\rightarrow} \Lambda^{0,1}(C, \mathcal{O}(n)) \rightarrow 0
$$

The metric $g_{\varphi}$, and the constant curvature metric of $C, g_{0}^{C}$, naturally induce metrics on $\Lambda^{0,0}(C, \mathcal{O}(N))$ and $\Lambda^{0,1}(C, \mathcal{O}(N))$. Hence, we define the adjoint of $\bar{\partial}, \bar{\partial}_{\varphi}^{*}$. Now we may define

$$
\Delta_{\varphi}=\bar{\partial} \bar{\partial}_{\varphi}^{*}+\bar{\partial}_{\varphi}^{*} \bar{\partial}: \Lambda^{0,0}(C, \mathcal{O}(N)) \rightarrow \Lambda^{0,0}(C, \mathcal{O}(N))
$$

to be the Laplacian operator with respect to the metric $g_{\varphi}$. Since $\Delta_{\varphi}$ is a well-defined elliptic operator, its regularized determinant can be defined by the method of zeta regularization. The following functional gives the difference of the determinant with respect to the conformal change of the metric:

\section{Definition 2.1.}

$$
A_{n}(\varphi)=\log \left(\frac{\operatorname{det} \Delta_{\varphi}}{\operatorname{det} \Delta_{0}}\right)
$$

In order to present $A_{n}$ more concretely, we consider the kernel of $\Delta$, which consists of all the polynomials of $z$ of degree at most $n$; thus, it is a complex linear space of dimension $n+1$ and has an othonormal basis with respect to the $g_{0}$ metric as follows:

$$
\alpha_{i}=\sqrt{(n+1)\left(\begin{array}{c}
n \\
i
\end{array}\right)} z^{i}, \quad i=0,1, \cdots, n .
$$

Because $C$ is one dimensional, by the Serre Duality, $A(\varphi)$ can also be viewed as analytic torsion associated to chain complex (2.4). Thus, by the the anomaly formula of Bismut-Gillet-Soulé [1], the following variational formula is obtained by Gillet-Soulé [3]:

\section{Proposition 2.2.}

$$
A_{n}(\varphi)=-\frac{1}{2} \int|\nabla \varphi|^{2} \mu-(n+1) \int \varphi \mu+\log \operatorname{det}\left(<\alpha_{i}, \alpha_{j}>_{\varphi}\right)_{n+1, n+1}
$$


Notice the similarity of $A_{n}$ with the left hand side of the Moser-Trudinger Inequality (2.2), which can be viewed as the log determinant of the scalar Laplacian on the sphere (without the involvement of the line bundle), and whose proof is related to the Yamabe problem of $S^{2}$.

It is a simple observation that the value of this functional is invariant when a constant function is added to $\varphi$; that is, for any constant $c \in \mathbb{R}$,

$$
A_{n}(\varphi)=A_{n}(\varphi+c)
$$

Hence, by subtracting a suitable constant, we may assume that $\int \varphi \mu=0$.

For future convenience, we define the following functional

$$
B_{n}(\varphi)=\log \operatorname{det}\left(<\alpha_{i}, \alpha_{j}>_{\varphi}\right)_{n+1, n+1},
$$

which is the $\mathrm{L}^{2}$ contribution of the anomaly formula; this is also the fully non-linear term of the functional $A_{n}$.

In [3], by a complicated generalization of original estimates of Moser, Gillet and Soulé proved the following

Theorem 2.3. (Gillet-Soulé) If $\varphi$ is rotationally symmetric (with respect to a given standard coordinate system on $C=S^{2}$ ), then

$$
B_{n}(\varphi) \leq\left(\frac{1}{2}-\varepsilon_{n}\right) \int|\nabla \varphi|^{2} \mu+(n+1) \int \varphi \mu+C_{n},
$$

where $\varepsilon_{n}>0$ and $C_{n}$ are constants depending only on $n$; in particular, $A_{n}(\varphi)$ is bounded by a constant independent of $\varphi$.

\section{Bounded-ness of the functional $A(\varphi)$.}

The main goal of this Section is to prove Theorem 1.2, which is a generalization of Theorem 2.3. Our strategy is to simplify the general case to the case which has been treated by Gillet and Soulé. Our approach involves symmetrization of various geometric quantities and their estimates, where the difficulty is to treat the highly non-linear term $B_{n}$.

First, we prove the following algebraic lemma.

Lemma 3.1. Let $d \lambda$ be a finite positive measure of space $C$. Let $0 \leq a_{0}<$ $\cdots \leq a_{n}$ be $n+1$ real numbers. For any $\sigma \in S_{n+1}$, define

$$
S_{\sigma, \lambda}=S_{\sigma}=\prod_{i=0}^{n} \int_{C}|z|^{a_{i}+a_{\sigma(i)}} d \lambda,
$$


then we have

$$
S_{\sigma} \leq S_{i d}
$$

Proof. This is just an extension of the Cauchy-Schwartz Inequality. We run an induction argument on $n$. The statement is obviously true for $n=1$, in which case it is just the usual Cauchy-Schwartz Inequality. Suppose it is true for $1, \cdots, n-1$. Fix a $\sigma \in S_{n}$. If $\sigma$ can be written as product of two shorter cycles, we can apply the induction hypothesis. Without loss of generality, we may assume

$$
\sigma=\left(0, i_{1}, i_{2}, \cdots, i_{n}\right) .
$$

To further simplify the notation, we assume that $a_{i}=i$. (Notice that the proof of general case follows similarly). Let

$$
\sigma^{\prime}=\left(i_{1}, i_{2}, \cdots, i_{n}\right)
$$

be a reduced $(n-1)$-cycle. Then we have,

$$
\log \left(S_{\sigma} / S_{\sigma^{\prime}}\right)=\log \int|z|^{i_{1}} d \lambda+\log \int|z|^{i_{n}} d \lambda-\log \int|z|^{i_{1}+i_{n}} d \lambda-\log \int 1 d \lambda .
$$

Simple calculation shows that

$$
u(t) \stackrel{\text { def }}{=} \log \int|z|^{i_{1}+i_{n}-t} d \lambda+\log \int|z|^{t} d \lambda
$$

is an increasing function for $t \in\left[0, \frac{i_{0}+i_{n}}{2}\right]$. Apply this fact to $0=t_{1}<t_{2}=$ $\min \left\{i_{1}, i_{n}\right\} \leq \frac{i_{1}+i_{n}}{2}$, we get

$$
S_{\sigma} \leq S_{\sigma^{\prime}}
$$

Now we can apply the induction hypothesis to prove that

$$
S_{\sigma} \leq S_{i d}
$$

Thus we have finished the induction proof.

Lemma 3.1 allows us to estimate the terms appearing in the determinant $B_{n}$ by the diagonal term. Now we state the main theorem of this section:

\section{Theorem 3.2.}

$$
B_{n}(\varphi) \leq\left(\frac{1}{2}-\varepsilon_{n}\right) \int|\nabla \varphi|^{2} \mu+(n+1) \int \varphi \mu+C_{n} ;
$$

where $\varepsilon_{n}>0$ and $C_{n}$ are constants depending only on $n$; in particular, $A(\varphi)$ is bounded from above by a constant independent of $\varphi$. 
Theorem 1.2 is then an easy corollary of Theorem 3.2.

From an analytic point of view, Theorem 3.2 can be viewed as a multiparticle Moser-Trudinger Inequality, generalizing the original inequality (2.2) of Moser and Trudinger [9]. We comment that the coefficient of the energy in (5.3) has a uniform upper bound independent of $n$, making it a very precise inequality. In the next sections, we will also discuss its sharp form.

Finally, we give the proof of Theorem 3.2.

Proof. First, by Lemma 3.1 and the definition of the determinant, we have

$$
\left|\operatorname{det}\left(<\alpha_{i}, \alpha_{j}>_{\varphi}\right)\right| \leq \sum_{\sigma \in S_{n+1}} S_{\sigma, \lambda} \leq n ! S_{i d, \lambda}
$$

where $d \lambda=\frac{\exp (\varphi)}{\left(1+|z|^{2}\right)^{n}} \mu$.

Notice that $l=\{z:|z|=1\}$ is a closed geodesic on $C$, with respect to the standard metric. Because of the rotational invariance of $C=S^{2}$, with out loss of generality, we assume that $\max _{z \in C} \varphi(z)$ is obtained at a point on $l$. Then, we discuss the rotational re-arrangement of $\varphi$, following [12]. Define $\varphi^{*}$ as the rotationally symmetric increasing re-arrangement of $\varphi$ for $|z| \leq 1$ and the rotationally symmetric decreasing re-arrangement function of $\varphi$ for $|z| \geq 1$. Hence, we have

$$
\begin{aligned}
\varphi^{*}(0) & =\min _{|z| \leq 1} \varphi(z), \\
\varphi^{*}(\infty) & =\min _{|z| \geq 1} \varphi(z), \\
\varphi(1) & =\max _{S^{2}} \varphi(z) .
\end{aligned}
$$

It is easy to see that $\varphi^{*}$ is continuous. By [12],

$$
\begin{aligned}
\int_{C}|\nabla \varphi|^{2} \mu & \geq \int_{C}\left|\nabla \varphi^{*}\right|^{2} \mu, \\
\int_{C} \varphi \mu & =\int_{C} \varphi \mu .
\end{aligned}
$$

Hence, $\varphi^{*} \in C^{0}(C) \cap W^{1,2}(C)$.

For $|z| \geq 1$, define, for $i=0, \cdots, n$,

$$
\begin{aligned}
P_{i} & =\frac{|z|^{2 i}}{\left(1+|z|^{2}\right)^{n}}, \\
P_{i}^{\prime} & =\frac{1}{\left(1+|z|^{2}\right)^{n-i}} .
\end{aligned}
$$


Then, for $|z| \geq 1$, we have

$$
P_{i} \leq P_{i}^{\prime} \leq 2^{2 i} P_{i} \leq 2^{2 n} P_{i} .
$$

Notice that both $P_{i}^{\prime}$ and $\varphi^{*}$ are non-increasing for $|z| \geq 1$, it is easy to check that

$$
\begin{aligned}
\log \int_{|z| \geq 1} P_{i} \exp (\varphi) \mu & \leq \log \int_{|z| \geq 1} P_{i}^{\prime} \exp (\varphi) \mu \leq \log \int_{|z| \geq 1} P_{i}^{\prime} \exp \left(\varphi^{*}\right) \mu \\
& \leq \log \int_{|z| \geq 1} P_{i} \exp \left(\varphi^{*}\right) \mu+2 n \log 2
\end{aligned}
$$

By the reflection symmetry of $C=S^{2}$ and the definition of $\varphi^{*}$, similarly we have

$$
\log \int_{|z| \leq 1} P_{i} \exp (\varphi) \mu \leq \log \int_{|z| \leq 1} P_{i} \exp \left(\varphi^{*}\right) \mu+2 n \log 2 .
$$

By (3.6) (3.10) and (3.11), we have, with $C(n)$ a constant depending only on $n$,

$$
\log \operatorname{det}\left(<\alpha_{i}, \alpha_{j}>_{\varphi}\right)_{n+1, n+1} \leq C(n)+\log \operatorname{det}\left(<\alpha_{i}, \alpha_{j}>_{\varphi *}\right)_{n+1, n+1} .
$$

Combine (3.7), (3.8) and (3.12), we have proved

$$
A(\varphi) \leq A\left(\varphi^{*}\right)+C(n) .
$$

Finally, by applying Theorem 2.3, we have proved the theorem.

\section{0 is a local maximum.}

Now that the functional $A_{n}$ is bounded by Theorem 3.2, it is natural to examine the sharp upper-bound of $A_{n}$. In this Section we show that the functional $A_{n}$ has a local maximal point at constant function, 0, for any fixed $n$. This stability result indicates that the maximum of functional $A_{n}$ should be achieved at the constant function.

We begin with some notations. For simplicity, we define the following local $(n+1) \times(n+1)$ matrices: for $\varphi, f \in C^{\infty}(C, \mathbb{R})$, write

$$
\begin{aligned}
\left(M_{\varphi}\right)_{i j} & =<\alpha_{i}, \alpha_{j}>_{\varphi}, \\
M_{\varphi}^{\prime}(f) & =<f \alpha_{i}, \alpha_{j}>_{\varphi}, \\
M_{\varphi}^{\prime \prime}(f) & =<f^{2} \alpha_{i}, \alpha_{j}>_{\varphi}, \\
\left(M_{\varphi}\right)^{i j} & =\left(M_{\varphi}\right)^{-1} .
\end{aligned}
$$

First, we give the following first variation formula: 
Lemma 4.1 (First Variation of $A_{n}$ ). If $g_{\varphi}$ is an extreme metric for the functional $A_{n}$, then

$$
\operatorname{tr}\left(\mathrm{M}_{\varphi}^{-1}\left[\frac{\alpha_{\mathrm{i}} \overline{\alpha_{\mathrm{j}}}}{\left(1+|\mathrm{z}|^{2}\right)^{\mathrm{n}}}\right]_{\mathrm{ij}}\right)+\Delta \varphi-(\mathrm{n}+1)=0 .
$$

In particular, the constant function 0 is a critical point of $A_{n}$ for any $n$.

Proof. The derivation of the above Euler-Lagrange equation is standard, which we omit here. To verify that 0 is critical, use the fact that $M_{0}=I d$, by (4.1), we only need to show

$$
n+1=\sum_{i=0}^{n} \frac{\alpha_{i} \cdot \overline{\alpha_{i}}}{\left(1+|z|^{2}\right)^{n}}
$$

or,

$$
n+1=\sum_{i=0}^{n} \frac{(n+1)\left(\begin{array}{c}
n \\
i
\end{array}\right)|z|^{2 i}}{\left(1+|z|^{2}\right)^{n}},
$$

which is obvious.

We then proceed to compute the second variation of $A_{n}$.

Lemma 4.2. For $\varphi, f \in C^{\infty}(C, \mathbb{R})$, the following holds

$$
\left.\frac{d^{2}}{d^{2} t}\right|_{t=0} A(\varphi+t f)=\operatorname{tr}\left[\mathrm{M}_{\varphi}^{-1} \mathrm{M}_{\varphi}^{\prime \prime}(\mathrm{f})\right]-\operatorname{tr}\left[\mathrm{M}_{\varphi}^{-1} \mathrm{M}_{\varphi}^{\prime}(\mathrm{f}) \mathrm{M}_{\varphi}^{-1} \mathrm{M}_{\varphi}^{\prime}(\mathrm{f})\right]-\int|\nabla \mathrm{f}|^{2} \exp \varphi \mu
$$

The proof of (4.3) is standard, so we omit it here.

Finally, we state the main result of this section.

Theorem 4.3. 0 is a local maximal point of the functional $A_{n}$.

Proof. By (4.3), we need to show the following inequality for any $f \in C^{\infty}(C)$,

$$
\sum_{i} \int_{C} f^{2} \alpha_{i j} \mu-\sum_{i, j}\left|\int_{C} f \alpha_{i j} \mu\right|^{2} \leq \int_{C}|\nabla f|^{2} \mu,
$$

where, for notational simplicity, we denote

$$
\alpha_{i j}=\frac{\alpha_{i} \overline{\alpha_{j}}}{\left(1+|z|^{2}\right)^{n}}
$$


Using the fact that $\left\{\alpha_{i}\right\}$ is an orthonormal basis, the left hand side of (4.4) can be re-written as

$$
\begin{aligned}
& \iint_{C \times C} \sum_{i j}\left(f^{2}(x) \alpha_{i j}(x) \overline{\alpha_{i j}(y)}-f(x) f(y) \alpha_{i j}(x) \overline{\alpha_{i j}(y)}\right) \mu_{y} \mu_{x} \\
= & \iint_{C \times C} \frac{1}{2} \sum_{i, j} \alpha_{i j}(x) \overline{\alpha_{i j}(y)}(f(x)-f(y))^{2} \mu_{x} \mu_{y} \\
= & \iint_{C \times C} \frac{(n+1)^{2}}{2}(f(x)-f(y))^{2} \sum_{i, j}\left(\begin{array}{c}
n \\
i
\end{array}\right)\left(\begin{array}{c}
n \\
j
\end{array}\right) \frac{z_{x}^{i} \bar{z}_{x}^{j} z_{y}^{i} \bar{z}_{y}^{j}}{\left(1+\left|z_{x}\right|^{2}\right)^{n}\left(1+\left|z_{y}\right|^{2}\right)^{n}} \mu_{x} \mu_{y} \\
= & \iint_{C \times C} \frac{(n+1)^{2}}{2}(f(x)-f(y))^{2}\left(\frac{\left|1+z_{x} \overline{z_{y}}\right|^{2}}{\left(1+\left|z_{x}\right|^{2}\right)\left(1+\left|z_{y}\right|^{2}\right)}\right)^{n} \mu_{x} \mu_{y} \\
= & \int_{C \times C} \frac{(n+1)^{2}}{2}(f(x)-f(y))^{2} \cos ^{2 n}\left(\sqrt{\pi} d_{\mu}(x, y)\right) \mu_{x} \mu_{y},
\end{aligned}
$$

where $d_{\mu}(\cdot, \cdot)$ is the distance function of $C=S^{2}$ with respect to the metric $\mu$. To do the local computation, sometimes we switch to the standard metric of $S^{2} \subset \mathbb{R}^{3}$, which has radius one and volume form $d v=4 \pi \mu$. If we use $d(\cdot, \cdot)$ to denote the distance function with respect to the standard metric, then

$$
d=\sqrt{4 \pi} d_{\mu}
$$

We continue our computation. By (4.5), (4.4) is equivalent to the following

$$
\iint_{C \times C} \frac{(n+1)^{2}}{2}(f(x)-f(y))^{2} \cos ^{2 n}\left(\frac{d(x, y)}{2}\right) \mu_{x} \mu_{y} \leq \int|\nabla f|^{2} \mu .
$$

Let $d l$ be the induced measure on embedded curves in $C$ from $d v$. For fixed points $x, y \in C$, let $l(x, y)$ be the shortest geodesic connecting $x$ and $y$. Then, the following estimate holds

$$
\begin{aligned}
|f(x)-f(y)|^{2} & \leq\left(\int_{l(x, y)}|\nabla f(p)| \cdot|\cos \delta(p, x, y)| d l_{p}\right)^{2} \\
& \leq d(x, y) \int_{l(x, y)}|\nabla f(p)|^{2} \cos ^{2} \delta(p, x, y) d l_{p},
\end{aligned}
$$

where $\delta(p, x, y)$ denotes the angle between the direction of $l(x, y)$ and $\nabla f(p)$. Obviously, for generic $(p, x, y), \delta$ can be determined only by $p$ and $x$; hence, it can also be written as $\delta(p, x)$. 
Fix $x \in C$ and write $\mu_{y}=\frac{1}{4 \pi} \sin \theta d \theta d \varphi$, with $\theta=d(x, y), \varphi \in[0,2 \pi), \theta \in$ $[0, \pi]$ being the spherical coordinates. Hence, if $l=d(p, x)$,

$$
\begin{aligned}
& \iint_{C \times C}|f(x)-f(y)|^{2} \cos ^{2 n}\left(\frac{d(x, y)}{2}\right) \mu_{x} \mu_{y} \\
\leq & \int_{x} \frac{1}{4 \pi} \int_{0}^{2 \pi} \int_{0}^{\pi} \cos ^{2 n}\left(\frac{\theta}{2}\right) \theta \sin \theta \int_{0}^{\theta}|\nabla f(p)|^{2} \cos ^{2} \delta(p, x) d l_{p} d \theta d \varphi \mu_{x} \\
= & \frac{1}{4 \pi} \int_{x} \int_{0}^{2 \pi} \int_{0}^{\pi}\left(\int_{l}^{\pi} \cos ^{2 n}\left(\frac{\theta}{2}\right) \theta \sin \theta d \theta\right) \cos ^{2} \delta(p, x)|\nabla f(p)|^{2} d l d \varphi \mu_{x} .
\end{aligned}
$$

Thus, if we define

$$
\left.G(l)=\int_{l}^{\pi} \cos ^{2 n}\left(\frac{\theta}{2}\right) \theta \sin \theta d \theta\right)
$$

The last expression of (4.9) can be written as

$$
\begin{aligned}
& \frac{1}{4 \pi} \int_{x} \int_{0}^{2 \pi} \int_{0}^{\pi} \cos ^{2} \delta(x, p)|\nabla f(p)|^{2} \frac{G(l)}{\sin l} \sin l d l d \varphi \mu_{x} \\
= & \frac{1}{4 \pi} \int_{x} \int_{p} \int_{0}^{\pi} \cos ^{2} \delta(x, p)|\nabla f(p)|^{2} \frac{G(l)}{\sin l} \mu_{p} \mu_{y} \\
= & \int_{p}|\nabla f(p)|^{2} \mu_{p}\left(\int \frac{G(d(x, p))}{\sin d(x, p)} \cos ^{2} \delta(x, p) \mu_{x}\right) .
\end{aligned}
$$

Hence, to prove (4.4), it is sufficient to prove the following

$$
\frac{(n+1)^{2}}{2} \int \frac{G(d(x, p))}{\sin d(x, p)} \cos ^{2} \delta(x, p) \mu_{x}<1 .
$$

We parameterize $x \in C$ by $l=d(x, p)$ and $\delta=\delta(x, p)$, such that $\mu_{x}=$ $\frac{1}{4 \pi} \sin l d l d \delta$. Hence, the left hand side of (4.12) equals

$$
\begin{aligned}
\frac{(n+1)^{2}}{2}\left(\frac{1}{4} \int_{0}^{\pi} \frac{G(l)}{\sin l} \sin l d l\right) & =\frac{(n+1)^{2}}{8} \int_{0}^{\pi} G(l) d l \\
& =\frac{(n+1)^{2}}{8} \int_{0}^{\pi} \cos ^{2 n}\left(\frac{\theta}{2}\right) \theta^{2} d \theta .
\end{aligned}
$$

By a change of variable $t=\frac{\theta}{2}$ and use the integration by part trick, we get

$$
\frac{(n+1)^{2}}{8} \int_{0}^{\pi} \cos ^{2 n}\left(\frac{\theta}{2}\right) \theta^{2} d \theta=2(n+1) \int_{0}^{\frac{\pi}{2}} t \cos ^{2 n+2} t d t .
$$


Define

$$
J_{n}=2(n+1) \int_{0}^{\frac{\pi}{2}} t \cos ^{2 n+2} t d t .
$$

Then (4.12) is equivalent to the following claim:

\section{Claim 4.4.}

$$
J_{n}<1, \quad \text { for } n \in \mathbb{N} \text {. }
$$

Proof. By integration by part, it is easy to get the following recursive formula

$$
J_{n}=-\frac{1}{2 n+2}+\frac{2 n+1}{2 n} J_{n-1} .
$$

To prove the claim, we make the following statement

$$
J_{n-1} \geq \frac{3 n+2}{3 n+3} .
$$

Obviously $(*)$ is wrong for $n=1$. If this is the case for all $n$, then the claim is proved. Otherwise, let $N$ be the smallest positive integer such that $\left(^{*}\right)$ is true. Then, by (4.14),

$$
\begin{aligned}
J_{N}-\frac{3 N+5}{3 N+6} & \geq-\frac{1}{2 N+2}+\left(\frac{2 N+1}{2 N}\right) \frac{3 N+2}{3 N+3}-\frac{3 N+5}{3 N+6} \\
& =\frac{4}{6 N(N+1)(N+2)}>0 .
\end{aligned}
$$

Thus, easy to show by induction that $(*)$ holds for all $n \geq N$. Therefore, by $(4.14)$,

$$
\begin{aligned}
J_{n}-J_{n-1} & =\frac{J_{n-1}}{2 n}-\frac{1}{2 n+2} \geq \frac{3 n+2}{(2 n)(3 n+3)}-\frac{1}{2 n+2} \\
& =\frac{1}{3 n(n+1)}>0
\end{aligned}
$$

in other words, by induction, $J_{n}$ is monotone increasing for $n \geq N$.

On the other hand, applying a standard stationary phase argument to (4.13), it is easy to see that

$$
\lim _{n \rightarrow \infty} J_{n}=1 .
$$

Hence, for $n<N, J_{n} \leq \frac{3 n+2}{3 n+3}<1$; for $n \geq N, J_{n} \leq J_{n+1} \leq \cdots<1$. The claim is proved. 
In summary, we have finished the proof of Theorem 4.3. It is also clear from (4.8) that the equality holds if and only if $|\nabla f|=0$ almost everywhere; i.e., $f$ is a constant function.

\section{Conjectured sharp inequality and its different formulations.}

Inspired by earlier computations, we pose the sharp inequality of our problem, and point out its links to different classical results.

\subsection{Conjecture on the sharp 2-D Szegö problem.}

We now discuss the classical Szegö limit theorem. Let $z$ be the complex coordinate function on $\mathbb{C}, S^{1} \subset \mathbb{C}$ be the standard circle of radius 1 , and $d s$ be the regular unit length measure on $S^{1}$. For a function $\varphi \in C^{\infty}\left(S^{1}, \mathbb{R}\right)$, define

$$
\begin{gathered}
\alpha_{i j} \stackrel{\text { def }}{=} z^{i} \bar{z}^{j}=z^{i-j} \\
B_{n}\left(\varphi, S^{1}\right) \stackrel{\text { def }}{=} \log \operatorname{det}\left[\int_{S^{1}} \alpha_{i j}(\exp \varphi) d s\right]_{(n+1) \times(n+1)} .
\end{gathered}
$$

As a consequence of the strong Szegö Limit Theorem, Szegö showed:

Theorem 5.1. $B_{n}\left(\varphi, S^{1}\right)$ is non-decreasing with respect to $n$. Furthermore,

$$
B_{n}\left(\varphi, S^{1}\right) \leq \frac{1}{2} \int|\nabla \varphi|^{2} d s+(n+1) \int \varphi d s .
$$

Equality holds iff $\varphi$ is a constant.

Noticing the similarity between (5.1) and (5.2) with our current problem, we pose the following conjecture for the standard two sphere:

Conjecture 5.2. For any $\varphi \in L^{2}\left(S^{2}, \mathbb{R}\right)$, we have

$$
A_{n}(\varphi) \leq 0
$$

or, equivalently,

$$
B_{n}(\varphi) \leq \frac{1}{2} \int|\nabla \varphi|^{2} \mu+(n+1) \int \varphi \mu
$$

equality holds iff $\varphi$ is a constant function. 
Conjecture 5.2 equivalent to Conjecture 1.3.

Remark 5.3. The coefficient of the energy in (5.3) is twice that of (2.2), the original Moser-Trudinger inequality. Thus, as pointed out by Gillet and Soulé, the above conjecture is true for $n=0,1$.

Remark 5.4. By Theorem 3.2, Conjecture 5.2 holds for $\varphi$ with large energy for general $n$. It also holds for $\varphi$ with very small energy by Theorem 4.3.

Remark 5.5. Due to the non-linear nature of our problem, for fixed $\varphi$, it is not clear if $B_{n}(\varphi)$ is monotone with respect to $n$. Notice that monotonicity is the key ingredient in Szegö's approach of Theorem 5.1.

Remark 5.6. Okikiolu extended a weak form of Szegö limit theorem to $S^{2}$ and $S^{3}$. In [10], she proved similar asymptotic results as that of Szegö for the determinant of the Toeplitz operator $M_{\exp \varphi}$ (see the next subsection for the notation) restricted to spherical harmonics of degree less than or equal to $n$. See Guillemin-Okikiolu [5] for an extension to the Zoll manifolds and Okikiolu [11] for a survey with further results and the probabilistic intepretation. However, Conjecture 5.2 is a holomorphic extension, in which we consider only the "holomorphic" part of the spherical harmonics.

This conjecture can also be viewed as the sharp form of the multi-particle Moser-Trudinger Inequality proved in Theorem 3.2. Actually, the proof of the original (sharp) Moser-Trudinger Inequality goes as follows: First, prove that an analogue of Theorem 1.2 holds; i.e., the functional has a universal upper bound. Then, get the sharp upper bound by analysis the Euler equation as a PDE. However, this strategy is hard to follow to prove Conjecture 5.2 since the Euler equation (4.1) for our multi-particle problem is highly non-linear and is not even a PDE.

\subsection{Toeplitz operator point of view.}

The functional $B_{n}$ has appeared in classical analysis in another form. Boutet de Monvel and Guillemin have considered the quantization problem for the Toeplitz operator in the framework of the pseudo-differential operator [2].

Let $F=\exp \varphi$. Let the Toeplitz operator $M_{F}$ be the multiplication operator with respect to $F . M_{F}$ acting on $\Gamma(\mathcal{O}(n))$ can be viewed as a quantization of $F$ :

$$
M_{F}=F \cdot: \Gamma(\mathcal{O}(n)) \rightarrow \Gamma(\mathcal{O}(n))
$$


Since $\left\{\alpha_{i}\right\}_{i=0}^{n}$ forms a basis of $\Gamma(\mathcal{O}(n))$,

$$
B_{n}(\varphi)=\log \operatorname{det} \int_{C}<M_{F} \alpha_{i}, \alpha_{j}>\mu
$$

is the so-called Toeplitz determinant. A special case of a theorem of Boutet de Monvel-Guillemin and a result of Uribe can be stated as follows:

Theorem 5.7. 1. (Boutet de Monvel-Guillemin) For $n>>1, B_{n}(\varphi)$ has the following asymptotic expansion:

$$
B_{n}(\varphi)=(n+1) \int_{C} \varphi \mu+\sum_{i=1}^{N} \frac{D_{i}(\varphi)}{(n+1)^{i}}+\frac{\widetilde{D_{N+1}}(n, \varphi)}{(n+1)^{N+1}},
$$

where $N \in \mathbb{N}, D_{i}(\varphi)$ 's are functionals independent of $n$ and dependent of $\varphi$ only, $\widetilde{D_{N+1}}(n, \varphi)$ is bounded independent of $n$;

2. (Uribe, [13]) furthermore,

$$
D_{0}(\varphi)=\frac{1}{2} \int_{C}|\nabla \varphi|^{2} \mu
$$

Theorem 5.7 shows that Conjecture 5.2 , with a fixed $\varphi$, is asymptotically correct for $n>>1$.

Notice that Uribe also gave an inductive method to compute all the $D_{i}(\varphi)^{\prime}$ s. However, it is not clear that the complete asymptotic expansion would lead to the sharp Moser Inequality.

\subsection{Probability formulation of functional $\mathbf{B}_{n}$.}

Using symmetries of $C=\mathbb{C P}^{1}=S^{2}$, we can reformulate the problem and give it a probabilistic interpretation. More precisely, we prove the following

Proposition 5.8. For $\left(z_{0}, \cdots, z_{n+1}\right) \in C^{n+1}$, define

$$
K_{n}\left(z_{0}, \cdots, z_{n+1}\right)=\frac{(n+1)^{n+1}\left(\begin{array}{l}
n \\
0
\end{array}\right) \cdots\left(\begin{array}{l}
n \\
n
\end{array}\right)}{(n+1) !} \prod_{0 \leq i<j \leq n}\left(\sin \frac{d\left(z_{i}, z_{j}\right)}{2}\right)^{2} .
$$

Then,

$$
\begin{aligned}
\int_{C^{n+1}} K_{n} \prod \mu_{z_{i}} & =1 \\
B(\varphi) & =\log \int_{C^{n+1}} \exp \left(\varphi\left(z_{0}\right)+\cdots+\varphi\left(z_{n}\right)\right) K_{n} \prod \mu_{z_{i}} .
\end{aligned}
$$


Proof. It is easy to see that (5.5) follows from (5.6) (by taking $\varphi=0$ ). Thus, we only need to prove the latter. For simplicity, we write $\mu_{i}=\mu_{z_{i}}$. Notice

$$
\begin{aligned}
\exp B(\varphi) & =\operatorname{det}\left[\int_{\mathbb{C}} \alpha_{i j} \exp \varphi \mu\right] \\
& =\frac{(n+1)^{n+1}\left(\begin{array}{l}
n \\
0
\end{array}\right) \cdots\left(\begin{array}{c}
n \\
n
\end{array}\right)}{(n+1) !} \int_{C^{n+1}} \exp \left(\sum \varphi\left(z_{i}\right)\right) \frac{\sum_{\sigma \in S_{n}}(-1)^{|\sigma|} \prod z_{i}^{i} \bar{z}_{i}^{\sigma(i)}}{\prod\left(1+\left|z_{i}\right|^{2}\right)^{n}} \prod \mu_{i} \\
& =\frac{(n+1)^{n+1}\left(\begin{array}{l}
n \\
0
\end{array}\right) \cdots\left(\begin{array}{c}
n \\
n
\end{array}\right)}{(n+1) !} \int_{C^{n+1}} \exp \left(\sum \varphi\left(z_{i}\right)\right) \frac{\prod_{i<j}\left|z_{i}-z_{j}\right|^{2}}{\prod\left(1+\left|z_{i}\right|^{2}\right)^{n}} \prod \mu_{i} .
\end{aligned}
$$

(5.6) then will be obvious considering the following simple fact on $S^{2}$ :

$$
\frac{\left|z_{i}-z_{j}\right|^{2}}{\left(1+\left|z_{i}\right|^{2}\right)\left(1+\left|z_{j}\right|^{2}\right)}=\sin ^{2} \frac{d\left(z_{i}, z_{j}\right)}{2} .
$$

We have finished the proof.

From Proposition 5.8, our study of the functionals $A$ and $B$ can be viewed probabilistically. Considering the $(n+1)$-random variables $\left\{z_{i}\right\}_{i=0}^{n}$ on the standard sphere $S^{2}$, with joint distribution given by $K_{n}$,

$$
\exp B_{n}(\varphi)=\mathbb{E}\left(\exp \left(\sum \varphi\left(z_{i}\right)\right)\right.
$$

is the expectation of the random variable $\exp \left(\sum \varphi\left(z_{i}\right)\right)$. Hence, for $n$ very large, our Conjecture 5.2 can also be related to a limit theorem of the given probability problem. This direction is partly motivated by the works of Kac [8] and Johansson [6] on the probabilistic approach to the Szegö limit theorem.

\section{References.}

[1] J.-M. Bismut, H. Gillet, and C. Soulé. Analytic torsion and holomorphic determinant bundles. I. Bott-Chern forms and analytic torsion. Comm. Math. Phys., 115(1):49-78, 1988.

[2] L. Boutet de Monvel and V. Guillemin. The spectral theory of Toeplitz operators, volume 99 of Annals of Mathematics Studies. Princeton University Press, Princeton, NJ, 1981. 
[3] H. Gillet and C. Soulé. Upper bounds for regularized determinants. Comm. Math. Phys., 199(1):99-115, 1998.

[4] Ulf Grenander and Gabor Szegö. Toeplitz forms and their applications. California Monographs in Mathematical Sciences. University of California Press, Berkeley, 1958.

[5] V. Guillemin and K. Okikiolu. Spectral asymptotics of Toeplitz operators on Zoll manifolds. J. Funct. Anal., 146(2):496-516, 1997.

[6] Kurt Johansson. On Szegő's asymptotic formula for Toeplitz determinants and generalizations. Bull. Sci. Math. (2), 112(3):257-304, 1988.

[7] Jürgen Jost and Guofang Wang. Analytic aspects of the Toda system. I. A Moser-Trudinger inequality. Comm. Pure Appl. Math., 54(11):12891319, 2001.

[8] M. Kac. Toeplitz matrices, translation kernels and a related problem in probability theory. Duke Math. J., 21:501-509, 1954.

[9] J. Moser. A sharp form of an inequality by N. Trudinger. Indiana Univ. Math. J., 20:1077-1092, 1970/71.

[10] Kate Okikiolu. The analogue of the strong Szegö limit theorem on the 2- and 3-dimensional spheres. J. Amer. Math. Soc., 9(2):345-372, 1996.

[11] Kate Okikiolu. High frequency cut-offs, trace formulas and geometry. In Spectral problems in geometry and arithmetic (Iowa City, IA, 1997), volume 237 of Contemp. Math., pages 107-121. Amer. Math. Soc., Providence, RI, 1999.

[12] Richard Schoen and S.-T Yau. Lectures on Differential Geometry, volume 1 of Conference Proceedings and Lecture Notes in Geometry and Topology. International Press, 1994.

[13] Alejandro Uribe. A symbol calculus for a class of pseudodifferential operators on $S^{n}$ and band asymptotics. J. Funct. Anal., 59(3):535-556, 1984.

Courant Institute of Mathematical Sciences

NeW York UNIVERSITY

RECEIVED JANUARY 21, 2004. 
